\title{
Genus Calculations of Complete Intersections
}

\author{
Feza Arslan and Sinan Sertöz \\ Bilkent University \\ Department of Mathematics
}

Communications in Algebra, 26(8) (1998), 2463-2471.

\section{Introduction}

In this article we calculate the genus of a projective complete intersection of any dimension and the genus of an affine complete intersection curve of superelliptic type. The arithmetic genus of a projective complete intersection depends on the degrees of the hypersurfaces cutting it. A formula for its genus using only the degrees of these hypersurfaces is given by Hirzebruch in [3] as an application of his Riemann-Roch theorem. Miranda has communicated us the following algorithm [5]:

One has to compute the cohomology $H^{i}\left(\mathcal{O}_{X}(k)\right)$ for every complete intersection $X \subset P^{n}$, for every twist $k$, and for every cohomology group $H^{i}$. Start with $X=P^{n}$ itself, where all is known. Assume you know everything when Y is a complete intersection with degrees $\left(d_{1}, d_{2}, \ldots, d_{r}\right)$ and let $X$ be a complete intersection with degrees $\left(d_{1}, d_{2}, \ldots, d_{r}, d_{r+1}\right)$. Hence $X$ is the intersection of $Y$ with a hypersurface of degree $d_{r+1}$. Then use the exact sequence

$$
0 \longrightarrow O_{Y}\left(k-d_{r+1}\right) \longrightarrow O_{Y}(k) \longrightarrow O_{X}(k) \longrightarrow 0 .
$$

Knowing the cohomology on the left and the middle will tell you the cohomology on the right. Applying this recursive algorithm with $X$ a curve gives the genus computation as $g=h^{1}\left(O_{X}\right)$.

We calculate the Hilbert polynomial of a projective complete intersection by using the Koszul resolution. The arithmetic genus is then obtained from the constant term of Hilbert polynomial. 
In the affine case the usual procedure for finding the genus of a curve is to find the genus of a resolution of its projective closure. This is intractable in general. We restrict our attention to the types of curves which are used in coding theory. These are superelliptic curves which we describe in equation (5). Stepanov and Özbudak has calculated the genus of some elliptic type curves in [9] using the "counting the differentials" method (for an account of this, see [7]). Stepanov also calculates the genus of an important class of curves in [8], see Remark 1 after Theorem (4) at the end of this article. Özbudak has also calculated the genera of fibre product of superelliptic curves using the "counting the differentials" and the Riemann-Hurwitz genus formula [6].

Khovanskii has given a formula for the arithmetic genus of an algebraic variety $X$ defined in $(\mathbb{C}-0)^{n}$ by a nondegenerate system of polynomial equations $f_{1}=\ldots=f_{k}=0$ with polyhedra $\Delta_{1}, \ldots, \Delta_{k}$. His method depends on choosing a toral nonsingular compactification $M^{n}$ of $(\mathbb{C}-0)^{n}$ and using an exact sequence of sheaves and cohomology groups of $M^{n}$ [4].

Our method involves describing the projective closure. After describing the ramification indices contributed by resolving the singular points at infinity, a direct application of the Riemann-Hurwitz formula yields the genus as a function of the degrees of the hypersurfaces cutting the curve. We also show how to count the regular differential on the curve.

Our main results are Theorem (1) and Corollary (2) for the projective case and Theorem (4) and Corollary (5) for the affine cases.

We thank R. Miranda, A. Klyachko and S. Stepanov for several discussions during the preparation of this work. The first author also would like to thank W. Vasconcelos, TÜBITTAK and Rutgers University Department of Mathematics for their generous support and hospitality during his visit.

\section{Projective Case}

Let $S$ denote the homogeneous coordinate ring, $k\left[x_{0}, \ldots, x_{n}\right]$, of $\mathbb{P}_{k}^{n}$, where $k$ is an algebraically closed field, usually $\mathbb{C}$. We assume that there are hypersurfaces $H_{1}, \ldots, H_{r}$ of $\mathbb{P}_{k}^{n}$ of degrees $d_{1}, \ldots, d_{r}$ respectively such that $X_{r}=H_{1} \cap \cdots \cap H_{r}$. The hypersurfaces $H_{1}, \ldots, H_{r}$ correspond to homogeneous polynomials $f_{1}, \ldots, f_{r} \in S$ of degrees $d_{1}, \ldots, d_{r}$ respectively. 


\subsection{The Hilbert Polynomial of $X_{r}$}

Theorem 1 The Hilbert polynomial $H_{r}(z)$ of $X_{r}$ is given by the following formula

$$
H_{r}(z)=\varphi(z)+\sum_{m=1}^{r}(-1)^{m} \sum_{1 \leq i_{1}<\cdots<i_{m} \leq r} \varphi\left(z-d_{i_{1}}-\cdots-d_{i_{m}}\right)
$$

where

$$
\varphi(z)=\frac{1}{n !}(z+1)(z+2) \cdots(z+n)=\left(\begin{array}{c}
z+n \\
n
\end{array}\right) .
$$

Proof: From [1, Theorem 2], the Koszul complex $K\left(f_{1}, \ldots, f_{r}\right)$ (which is the Koszul complex of the homomorphism $\varphi: S^{r}=\oplus_{i=1}^{r} S e_{i} \rightarrow S=k\left[x_{0}, \ldots, x_{n}\right]$ such that $\varphi\left(e_{i}\right)=f_{i}$ for $\left.1 \leq i \leq r\right)$ is a free resolution of $S /\left(f_{1}, \ldots, f_{r}\right)$. Namely, we have the following exact sequence

$$
0 \rightarrow \wedge^{r}\left(S^{r}\right) \rightarrow \ldots \rightarrow \wedge^{2}\left(S^{r}\right) \rightarrow S^{r} \rightarrow S \rightarrow S /\left(f_{1}, \ldots, f_{r}\right) \rightarrow 0 .
$$

In $[2]$, in order to grade

$$
\wedge^{m}\left(S^{r}\right)=\bigoplus_{1 \leq i_{1}<\ldots<i_{m} \leq r} S e_{i_{1}} \wedge \ldots \wedge e_{i_{m}}(1 \leq m \leq r)
$$

a degree $d_{i_{1}}+\ldots+d_{i_{m}}$ is assigned to a basis element $e_{i_{1}} \wedge \ldots \wedge e_{i_{m}}$, so that (2) is an exact sequence with maps homogeneous of degree zero. Now imposing the additive property of Hilbert polynomials on the exact sequence (2), the formula given in (1) is obtained.

Corollary 2 The arithmetic genus, $g_{a}\left(X_{r}\right)$, of $X_{r}$ is given by the formula

$$
g_{a}\left(X_{r}\right)=\sum_{m=1}^{r}(-1)^{m+n-r} \sum_{1 \leq i_{1}<\cdots<i_{m} \leq r} \varphi\left(-d_{i_{1}}-\cdots-d_{i_{m}}\right) .
$$




\section{Affine Case}

In this section, we compute the genus of a complete intersection curve $C$ in $\mathbb{A}_{\mathbb{C}}^{n+1}$ given by,

$$
\begin{aligned}
y_{1}^{d_{1}}= & \left(x-a_{11}\right) \ldots\left(x-a_{1 m}\right) \\
y_{2}^{d_{2}}= & \left(x-a_{21}\right) \ldots\left(x-a_{2 m}\right) \\
\vdots & \vdots \\
y_{n}^{d_{n}}= & \left(x-a_{n 1}\right) \ldots\left(x-a_{n m}\right)
\end{aligned}
$$

where $2 \leq d_{1} \leq \cdots \leq d_{n} \leq m-1$ and all $a_{i j}$ 's are distinct, with $a_{i j} \in \mathbb{C}$.

This is a smooth affine curve and its projective closure $\bar{C}$ in $\mathbb{P}_{\mathbb{C}}^{n+1}$ is singular. Let $\tilde{C}$ be a resolution of $\bar{C}$. The genus of $C$ is then defined as the genus of $\tilde{C}$. In the following subsections we will in turn describe the projective closure of $C$, describe a finite map from $\tilde{C}$ to $\mathbb{P}^{1}$, count the ramification indices of the points of $\tilde{C}$ under this map and finally apply the Riemann-Hurwitz formula to this map to calculate the genus.

\subsection{A Finite Morphism to $\mathbb{P}^{1}$}

In order to compute the genus of a nonsingular model $\tilde{C}$ of the projective closure $\bar{C}$ of $C$ we first define a finite morphism from $\tilde{C}$ to $\mathbb{P}^{1}$.

There exists a finite morphism

$$
\begin{aligned}
\varphi: C & \rightarrow \mathbb{C} \\
\left(x, y_{1}, \ldots, y_{n}\right) & \mapsto x
\end{aligned}
$$

$C$ is embedded into $\mathbb{P}^{n+1}$ the same way $\mathbb{C}$ embeds into $\mathbb{P}^{1}$. The morphism $\varphi$ extends to $\bar{C}$ algebraically by defining

$$
\begin{aligned}
\varphi: \bar{C} & \rightarrow \mathbb{P}^{1} \\
{\left[x: y_{1}: \cdots: y_{n}: 1\right] } & \mapsto[x: 1] \\
{\left[0: y_{1}: \cdots: y_{n}: 0\right] } & \mapsto[1: 0]
\end{aligned}
$$

See also the parametrization (8) for a justification of this definition. If $\tilde{C}$ is a resolution of $\bar{C}$, then $C$ and $\tilde{C}$ are isomorphic everywhere except at finitely many points which correspond to the points at infinity and $\varphi$ extends over to $\tilde{C}$ by sending all the points at infinity to $[1: 0]$ as above. 
Thus we have a map

$$
\varphi: \tilde{C} \rightarrow \mathbb{P}^{1}
$$

which is a morphism of degree $d_{1} d_{2} \ldots d_{n}$.

\subsection{Ramifications of $\varphi$}

We first examine the $n=2$ case with $d=d_{1}=d_{2}$. Consider the curve $C_{1}$ given by the equations

$$
\begin{aligned}
& y_{1}^{d}=x^{m}+a_{1} x^{m-1}+\ldots+a_{m-1} x+a_{m}=: F_{1}(x) \\
& y_{2}^{d}=x^{m}+b_{1} x^{m-1}+\ldots+b_{m-1} x+b_{m}=: F_{2}(x)
\end{aligned}
$$

with $2 \leq d \leq m-1$. For the points in the affine plane we can take $x$ as a local parameter. When $x$ is not equal to any of the $a_{i j}$ 's then the ramification of $\varphi$ at $x$ is 1 . When $x=a_{i j}$, then the ramification of $\varphi$ at $x$ is $d$. (For the general case of equation (5) the ramification at $a_{i j}$ is $d_{1} \cdots \hat{d}_{i} \cdots d_{n}$, where $\hat{d}_{i}$ denotes that the term should be omitted.)

To examine the points at infinity choose a local parameter $t$ with $x=1 / t$. Then we have

$$
\begin{aligned}
y_{1}^{d} & =x^{m}+a_{1} x^{m-1}+\cdots+a_{m-1} x+a_{m} \\
& =(1 / t)^{m}+a_{1}(1 / t)^{m-1}+\cdots+a_{m-1}(1 / t)+a_{m} \\
& =\left(1+a_{1} t+\cdots+a_{m-1} t^{m-1}+a_{m} t^{m}\right) / t^{m} .
\end{aligned}
$$

Let

$$
\begin{gathered}
d=a c \\
m=b c, \quad(a, b)=1, \quad c \geq 1 .
\end{gathered}
$$

Define a new local parameter $T$ such that

$$
T^{a}=t .
$$

Then the above parametrization of $y_{1}^{d}$ becomes

$$
y_{1}^{a c}=\left(1+a_{1} T^{a}+\cdots+a_{m} T^{a b c}\right) / T^{a b c} .
$$


Similarly we have

$$
y_{2}^{a c}=\left(1+b_{1} T^{a}+\cdots+b_{m} T^{a b c}\right) / T^{a b c} .
$$

Let $H_{1}(T)$ and $H_{2}(T)$ be power series such that $y_{1}^{a c}=H_{1}^{a c}\left(T^{a}\right) / T^{a b c}$ and $y_{2}^{a c}=H_{2}^{a c}\left(T^{a}\right) / T^{a b c}$. Then the points around infinity are parametrized as

$$
P\left(\alpha_{1}, \alpha_{2}, T\right)=\left[\frac{T^{b}}{\alpha_{1} T^{a} H_{1}\left(T^{a}\right)}: 1: \frac{\alpha_{2}}{\alpha_{1}} \frac{H_{2}\left(T^{a}\right)}{H_{1}\left(T^{a}\right)}: \frac{T^{b}}{\alpha_{1} H_{1}\left(T^{a}\right)}\right],
$$

where $\alpha_{1}$ and $\alpha_{2}$ are $d$-th roots of unity. Note that $H_{1}(0)=H_{2}(0)=1$ and thus the points at infinity are of the form $\left[0: 1: \alpha_{2} / \alpha_{1}: 0\right]$. In the $T$-plane let $T_{1}$ and $T_{2}$ be two points such that $T_{2}=\lambda T_{1}$ where $\lambda$ is an $a$-th root of unity. We have $T_{1}^{a}=T_{2}^{a}$ but $T_{1}^{b} \neq T_{2}^{b}$ since $(a, b)=1$. Hence $P\left(\alpha_{1}, \alpha_{2}, T_{1}\right) \neq$ $P\left(\alpha_{1}, \alpha_{2}, T_{2}\right)$. As $T$ ranges in the $T$-plane $P\left(\alpha_{1}, \alpha_{2}, T\right)$ describes a branch of the curve at infinity. There are then $d^{2} / a=d c$ branches at infinity. Since there are $d$ points at infinity, around each such point there are then $c$ branches making the total of $d c$ branches. Each branch corresponds to a different point on the resolution so there are $d c$ points on the resolution corresponding to the points at infinity, i.e. the cardinality of the set $\varphi^{-1}([1: 0]) \subset \tilde{C}$ is $d c$. Total ramification index for the preimage of any point under $\varphi$, i.e. the degree of $\varphi$, is $d^{2}$. This gives a ramification index of $a$ for each point in the resolution corresponding to the point at infinity.

In the general case when $d=d_{1}=\cdots=d_{n}$, the total ramification index of $\varphi$ is $d^{n}$, there are $d^{n-1} c$ branches at infinity each having ramification index $a$. This is the case for the curve define with the equations (9).

In the most general case, see equations (5), when $d=d_{1}=\cdots=d_{s}<$ $\cdots<d_{n}$ there are $d^{s-1} c$ branches at infinity each with ramification index $a d_{s+1} \cdots d_{n}$. In this case the cardinality of $\varphi^{-1}\left(\left[a_{i j}: 1\right]\right)$ is $d_{1} \cdots \hat{d}_{i} \cdots d_{n}$ and the ramification index of each such point is $d_{i}-1$. The total degree of $\varphi$ is $d^{s} d_{s+1} \cdots d_{n}$.

Before we apply the Riemann-Hurwitz formula to find the genus we summarize our observations about the points at infinity of the curve $C$ of equation (5) in the following corollary.

Corollary 3 Let $C$ be the curve in $\mathbb{A}^{n+1}$ defined by (5), with $d_{1} \leq d_{2} \leq$ $\ldots \leq d_{n}$. Assume that for some $s$ the first $s d_{i}$ 's are equal, i.e. $d=d_{1}=$ 
$d_{2}=\ldots=d_{s}<d_{s+1}<\ldots<d_{n}$. Then the projective closure $\bar{C}$ of the curve $C$ in $\mathbb{P}^{n+1}$ is the union of $C$ and the points of the form,

$$
\left[x: y_{1}: \cdots: y_{s}: y_{s+1}: \cdots: y_{n}: z\right]=\left[0: 1: \alpha_{2}: \cdots: \alpha_{s}: 0: \cdots: 0\right]
$$

where $\alpha_{2}^{d}=\ldots=\alpha_{s}^{d}=1$.

\subsection{The Genus Calculation}

The Riemann-Hurwitz formula for the map $\varphi$ takes the form

$$
\begin{aligned}
g_{C} & =1-\operatorname{deg} \varphi+\frac{1}{2} \sum_{x \in C}\left(e_{x}-1\right) \\
& =1-\operatorname{deg} \varphi+\frac{1}{2} \sum_{x \in \varphi^{-1}([*: 1])}\left(e_{x}-1\right)+\frac{1}{2} \sum_{x \in \varphi^{-1}([1: 0])}\left(e_{x}-1\right),
\end{aligned}
$$

where $e_{x}$ denotes the ramification index.

Theorem 4 Let $C$ be the complete intersection curve given by,

$$
\begin{aligned}
y_{1}^{d}= & \left(x-a_{11}\right) \ldots\left(x-a_{1 m}\right) \\
y_{2}^{d}= & \left(x-a_{21}\right) \ldots\left(x-a_{2 m}\right) \\
\vdots & : \\
y_{n}^{d}= & \left(x-a_{n 1}\right) \ldots\left(x-a_{n m}\right)
\end{aligned}
$$

where $d+1 \leq m$, and all $a_{i j}$ 's are distinct. The genus of $C$ is given by the formula

$$
g_{C}=1-\frac{1}{2}(d-m n d+m n+c) d^{n-1}
$$

where $c=(d, m)$.

Proof: The degree of $\varphi$ is $d^{n}$. The ramification index at smooth finite points is 1 and for each point $x \in C$ for which $\varphi(x)=a_{i j}$ the ramification 
index is $d$. There are $d^{n-1}$ points in $\varphi(x)=a_{i j}$ and the number of $a_{i j}$ 's is $m n$. This gives $\frac{1}{2} m n d^{n-1}(d-1)$ for the first summation in (10).

There are $d^{n-1} c$ points on the resolution of the projective closure of $C$ corresponding to points at infinity. Each such point has ramification index $a$. This then gives $\frac{1}{2} d^{n-1} c(a-1)$ for the second summation in (10). Putting these in and simplifying gives the seeked formula.

Remark 1: Putting in $d=2, c=1$ we recover Stepanov's formula $1+(m n-3) 2^{n-2}$, see $[8$, p370, Lemma 1]. Stepanov arrives at this formula by constructing an explicit basis for the differential forms of the curve. He works over a finite field $F_{q}$ of characteristic $p>2$.

We finally give the formula for the most general case.

Corollary 5 Let $C$ be the complete intersection curve given by (5), with $d_{1} \leq d_{2} \leq \ldots \leq d_{n}$ and with the first $s d_{i}$ 's equal to $d$. The genus of $C$ is given by the formula

$$
\begin{aligned}
g_{C}= & 1-\frac{1}{2}(d-m n d+m s) d^{s-1} d_{s+1} \cdots d_{n}-\frac{1}{2} d^{s-1} c \\
& -\frac{m d^{s}}{2} \sum_{i=s+1}^{n} d_{s+1} \cdots \hat{d}_{i} \cdots d_{n},
\end{aligned}
$$

where $c=(d, m)$.

Remark 2: This corollary can be proved in the same way as Theorem (4). The ramification values required for the formula are given at the end of section (3.2).

Remark 3: Note that when we put $s=n$ in the above formula (11) we recover the formula (10) of Theorem (4). However this is only an algebraic phenomena since geometrically the two formulas are derived from different configurations at infinity. 


\subsection{Counting the Differentials}

It is of interest to summarize the method of counting the regular differentials for the curve $C$ of equation (9). See also the equations (7) for the conventions in use. For any point in the affine space let $x$ be a local parameter and consider the regular 1-form

$$
\omega_{i_{1}, \ldots, i_{\sigma}}^{\left(j_{1}, \ldots, j_{\sigma}\right)}=\frac{d x}{y_{i_{1}}^{j_{1}} \cdots y_{i_{\sigma}}^{j_{\sigma}}}
$$

where $1 \leq \sigma \leq n, 1 \leq i_{1}<\cdots<i_{\sigma} \leq n$ and $1 \leq j_{1}, \ldots, j_{\sigma} \leq d-1$. By checking the order of vanishings of $x$ and $y_{i}$ 's it can be shown that the form $\omega_{i_{1}, \ldots, i_{\sigma}}^{\left(j_{1}, \ldots, j_{\sigma}\right)}$ is regular at any point in the affine space. Let $x_{\infty}$ be any point at infinity on the projective closure of $C$. Let $\nu_{\infty}$ denote the order of vanishing of a function at $x_{\infty}$. Choosing $t=1 / x$ as a local parameter around $x_{\infty}$ we observe that

$$
\begin{gathered}
\nu_{\infty}(x)=-a \\
\nu_{\infty}\left(y_{i}\right)=-b .
\end{gathered}
$$

Let $\bar{\omega}_{i_{1}, \ldots, i_{\sigma}}^{\left(j_{1}, \ldots, j_{\sigma}\right)}$ denote the expression for $\omega_{i_{1}, \ldots, i_{\sigma}}^{\left(j_{1}, \ldots, j_{\sigma}\right)}$ around $x_{\infty}$. We then have

$$
\nu_{\infty}\left(\bar{\omega}_{i_{1}, \ldots, i_{\sigma}}^{\left(j_{1}, \ldots, j_{\sigma}\right)}\right)=\left(j_{1}+\cdots+j_{\sigma}\right) b-a-1
$$

and if $P(x)$ is a polynomial then $P(x) \omega_{i_{1}, \ldots, i_{\sigma}}^{\left(j_{1}, \ldots, j_{\sigma}\right)}$ is regular at $x_{\infty}$ if and only if $\operatorname{deg} P(x) \leq\left(\left(j_{1}+\cdots+j_{\sigma}\right) b-a-1\right) / a$. We can then give a basis for the regular differential 1-forms;

$$
\begin{aligned}
\left\{x^{r} \omega_{i_{1}, \ldots, i_{\sigma}}^{\left(j_{1}, \ldots, j_{\sigma}\right)} \mid\right. & \sigma=1, \ldots, n, 1 \leq i_{1}<\cdots<i_{\sigma} \leq n \\
& 1 \leq j_{1}, \ldots, j_{\sigma} \leq d-1 \\
& \left.0 \leq r \leq\left(\left(j_{1}+\cdots+j_{\sigma}\right) b-a-1\right) / a\right\}
\end{aligned}
$$

The cardinality of this set then gives the genus of the curve $C$. It turns out that the required formula is

$$
g(C)=\sum_{\sigma=1}^{n} \sum_{1 \leq i_{1}<\cdots<i_{\sigma} \leq n} \sum_{j_{1}=1}^{d-1} \cdots \sum_{j_{\sigma}=1}^{d-1} \llbracket \frac{\left(j_{1}+\cdots+j_{\sigma}\right) b-1}{a} \rrbracket,
$$


where $\llbracket \llbracket$ denotes the greatest integer function. Note that this formula now works on any algebraically closed field of any characteristic, when $a \neq 0$.

Stepanov has calculated this sum for $d=2$ and $c=1$ over a field of characteristic $p>2$, [8, p372], (in that case $d=a=2$ and $m=b$ is odd).

The sceptic reader can check the validity of equation (12) using a computer; as equation (10) suggests the genus grows exponentially as $n$ increases so we suggest an example with a small $n$, for example $n=3$. Let $d=66$, $m=385$. Then $a=6, b=35$ and $c=11$. Putting these in equations (10) and (12) both gives the genus as 163345645 .

\section{References}

[1] Eagon, J. A., Northcott, D. G., Ideals defined by matrices and a certain complex associated with them, Proc. Roy. Soc. (A), 269 (1962), 188-204

[2] Eagon, J. A., Northcott, D. G., A Note on the Hilbert functions of certain ideals which are defined by matrices, Mathematika, 9 (1962), $118-126$

[3] Hirzebruch, F., Topological Methods in Algebraic Geometry, SpringerVerlag, 1978.

[4] Khovanskii, A. G., Newton polyhedra and the genus of complete intersections, Funkts. Anal. Prilozhen., No. 1 (1978), 51-61

[5] Miranda, R., Private communication.

[6] Özbudak, F., Codes on Fibre Products of Some Kummer Coverings, to appear in Finite Fields and Their Applications.

[7] Shafarevich, I., R., Basic Algebraic Geometry I, Springer-Verlag, 1977

[8] Stepanov, S., Character sums and coding theory, Finite Fields and Applications, (1996), 355-378.

[9] Stepanov, S. A., Özbudak, F.,Fibre Products of Hyperelliptic Curves and Geometric Goppa Codes, Discrete Math. Appl., 7 (1997), 223-229.

sarslan@fen.bilkent.edu.tr

sertoz@fen.bilkent.edu.tr 